\title{
Soleus muscle as a surrogate for health status in human heart failure?
}

\author{
Daniel J. Green ${ }^{1,2}$, Fausto A. Panizzolo ${ }^{1}$, David G. Lloyd ${ }^{1,3}$, Jonas Rubenson ${ }^{1,4}$, \\ Andrew J. Maiorana ${ }^{5,6,7}$ \\ ${ }^{1}$ The School of Sport Science, Exercise and Health, \\ The University of Western Australia, Crawley, WA, 6009, Australia \\ ${ }^{2}$ Research Institute for Sport and Exercise Science, \\ Liverpool John Moores University, Liverpool, United Kingdom \\ ${ }^{3}$ Centre for Musculoskeletal Research, \\ Menzies Health Institute Queensland \\ ${ }^{4}$ Biomechanics Laboratory, Department of Kinesiology, \\ Pennsylvania State University, University Park, PA, 16802, USA \\ ${ }^{5}$ Advanced Heart Failure and Cardiac Transplant Service, \\ Royal Perth Hospital, Perth, WA 6000, Australia \\ ${ }^{6}$ School of Physiotherapy and Exercise Science, \\ Curtin University, Perth, WA 6102, Australia \\ ${ }^{7}$ Allied Health Department, \\ Fiona Stanley Hospital, Murdoch, 6150 WA, Australia
}

Author for correspondence:

Winthrop Professor Daniel J. Green

The School of Sport Science, Exercise and Health, The University of Western Australia, Crawley, WA, 6009, Australia. Tel: + 6186488 5533, Email: danny.green@uwa.edu.au

SHORT TITLE: Skeletal muscle in chronic heart failure

DISCLOSURE AND CONFLICT OF INTEREST: No conflicts of interest, financial or otherwise, are declared by the authors.

\section{GRANTS}

This work was supported by a Grant-in-Aid (G09P 4469) from the National Heart Foundation of Australia to J.R., D.J.G., A.J.M. and D.G.L. and a thesis dissertation grant from the International Society of Biomechanics to F.A.P. 


\section{ABSTRACT}

We propose the hypothesis that soleus muscle function may provide a surrogate measure of functional capacity in patients with heart failure. We summarize literature pertaining to skeletal muscle as a locus of fatigue and present our recent findings, utilising in vivo imaging in combination with biomechanical experimentation and modelling, to reveal novel structure-function relationships in CHF skeletal muscle and gait.

\section{SUMMARY}

We propose the hypothesis that soleus function provides a surrogate for functional capacity and health status in chronic heart failure.

KEYWORDS: active force, passive force, strength, skeletal muscle, heart failure, exercise training 


\section{INTRODUCTION}

\section{A BRIEF HISTORY OF EXERCISE AND SKELETAL MUSCLE FUNCTION IN CHRONIC HEART FAILURE MANAGEMENT}

The 11th edition of Harrison's Textbook of Internal Medicine, published in 1987, stated that treatment of heart failure should "consist of reducing physical activity, instituting rest and reducing afterload.” By the early 2000's, the American Heart Association concluded that exercise training in chronic heart failure (CHF) was "safe and beneficial overall in improving peak $\dot{V} \mathrm{O}_{2}$, peak workload, exercise duration, and parameters of submaximal exercise performance. In addition, quality of life improves...." (27). $\quad$ This statement led other peak bodies to produce recommendations such as: $\quad$ "When medically stable, all CHF patients should be considered for referral to an exercise program" (22).

The narrative underlying this paradigm shift is an exemplar of translational research in exercise science, with ultimate global impact on patient management. The story has several starting points, including: recognition of the importance of peak oxygen uptake ( $\dot{\mathrm{V}} \mathrm{O}_{2}$ peak) as an index of systemic and integrated physiological function that outperforms traditional indices used in prognostication and transplant stratification (19), identification of skeletal muscle as an important locus of exercise intolerance in heart failure (8), courageous early exercise-training studies illustrating the reversibility of such limitations (31), ambitious studies indicating that training could induce improvement in outcomes (2) and establishment of the hemodynamic safety of well controlled and targeted exercise interventions (3). There are also early examples of patient-centred individualisation of exercise prescription utilising novel and targeted approaches $(15,17)$, a trend that is only now emerging as a mainstream agenda. 
In this brief paper, we revisit and acknowledge the important contributions made by studies that indicated that peripheral limitations to performance are amenable to modification in CHF. We also present some recent findings using contemporary techniques for the study of skeletal muscle function in vivo to pose the novel hypothesis that soleus muscle function may provide a valid surrogate measure for functional capacity and health status in patients with chronic heart failure.

\section{THE ONGOING BURDEN OF HUMAN CHRONIC HEART FAILURE}

Chronic heart failure continues to be a major global health burden. Two million new cases of heart failure are diagnosed worldwide every year, for an estimated total of 22 million affected. In the US, recent data indicate that 5.1 million people are affected by CHF and it is one of the most common reasons for hospital admission and general practitioner consultations in the elderly. Despite some improvement across recent decades, the mortality rates associated with CHF remain very high, with a reported risk-adjusted 1-year mortality of $29.6 \%$ in 2008 in the US (4).

\section{CLINICAL RELEVANCE OF THE LOCUS OF THE CLINICAL SYMPTOMS OF FATIGUE IN CHRONIC HEART FAILURE}

Poor exercise tolerance is a cardinal symptom of CHF, which significantly impacts upon quality of life and the capacity to perform tasks of daily living (6,9). Peak oxygen uptake outperforms ejection fraction and hemodynamic indices in terms of prognostication (19) and is used to guide transplantation and ventricular assist device implantation $(19,20)$.

Research over the past two decades has identified an intriguing paradox in CHF, in that the primary locus of exercise intolerance often lies in the skeletal muscle, rather than the heart itself $(7,26)$. Aerobic capacity and resting cardiac function are poorly correlated in patients with CHF (9). Instead, peripheral factors including impaired blood flow (17) and skeletal muscle dysfunction (6) 
have a greater bearing on aerobic capacity and are intrinsically linked to the exercise intolerance in this condition.

Recognition of the importance of peripheral abnormalities in CHF led to the "skeletal muscle hypothesis” (6), which proposes that activation of muscle ergoreceptors (receptors activated during muscle contractions that mediate physiologic adjustments) generate symptoms during exercise and that muscle wasting contributes to a wicked cycle of reflex neuro-hormonal activation, peripheral vasoconstriction, disease progression and physical inactivity (Figure 1). Consistent with the muscle hypothesis, muscle wasting is strongly correlated with reduced aerobic capacity (5) and poor prognosis (1). A recent analysis of $200 \mathrm{CHF}$ patients revealed that reduced muscle mass is independently associated with lower absolute $\dot{V} O_{2}$ peak $\left(\mathrm{ml} \cdot \mathrm{min}^{-1}\right)$ when age, sex, ejection fraction and co-morbidities were accounted for, and that muscle mass is directly linked to disease progression (11). These findings place skeletal muscle function at center-stage when exercise-based strategies are devised to target functional limitation in a large proportion of patients with stable CHF.

\section{SKELETAL MUSCLE ARCHITECTURE AND MECHANICS IN HUMAN CHRONIC HEART FAILURE}

Muscle architecture is closely linked to in vivo muscle mechanics. Muscle volume is one of the primary determinants of the muscle’s capacity to generate mechanical work. Maximal muscle force, and thus also joint torque production is, on the other hand, more closely associated with muscle physiological cross sectional area (PCSA) (29). PCSA is determined by muscle volume, as well as fibre length and pennation angle (25). Whilst some studies have examined muscle size in context of strength and functional limitations in CHF (12), few have investigated detailed architectural parameters. 
In addition, two mechanical properties that influence in vivo muscle mechanics independently of muscle volume and PCSA are its fiber operating lengths and velocities (force-length and forcevelocity properties). To the best of our knowledge no previous studies have examined fiber length and velocity characteristics of skeletal muscle in CHF. It is widely accepted that muscles operating outside their optimal length and velocity ranges are severely restricted in their ability to produce force, work and power, resulting in loss of function (29). The capacity of muscle to generate mechanical work is optimal when it functions over the plateau region of the force-length curve. When skeletal muscle operates outside this range (e.g. if muscle fiber lengths are reduced), mechanical work and power are drastically compromised. Although abnormalities in CHF skeletal muscle structure such as atrophy and a shift to type II fibers (6) and decreased cross-bridge kinetics (21) make them susceptible to sub-optimal function, muscle architecture and mechanics as a limitation to skeletal muscle function have not previously been studied in CHF.

\section{RECENT STUDIES RELATING PLANTARFLEXOR MUSCLE MORPHOLOGY AND FUNCTION TO EXERCISE CAPACITY IN CHRONIC HEART FAILURE}

We recently reported alterations on the functional and morphological properties of the plantarflexor muscles in patients affected by CHF (NYHA Class II-IV; ejection fraction < $40 \pm 10 \%$; age $62 \pm 10$ yr; mean \pm SD) (25), relative to control participants matched for age, sex, adiposity and physical activity levels. These studies are based on detailed measurements of muscle architecture using stateof-the art 3D ultrasound imaging (dynamic B-mode imaging coupled with simultaneous motion capture of the probe) and estimates of active and passive muscle loads from a novel framework incorporating empirical data (dynamic muscle fascicle imaging, electromyography and dynamometry) and musculoskeletal modeling. In an effort to maximize accuracy, the derived estimates of muscle loads took into account agonist and antagonist muscle actions, muscle-tendon dynamics and subject-specific muscle moment arm data (Figure 2). 
Despite a similar overall lower limb lean mass, CHF patients exhibited 25\% lower combined triceps surae volume (Fig. 3a) and physiological cross-sectional area (PCSA) (25). These reductions in triceps surae size were explained primarily by the marked reductions in the soleus (SOL). A strong correlation $(\mathrm{r}=0.93)$ was observed between absolute triceps surae volume and $\dot{\mathrm{V}} \mathrm{O}_{2}$ peak in CHF patients but not control participants. Furthermore, body mass-specific SOL volume, unlike the other triceps surae muscles, correlated strongly with body mass-specific $\dot{\mathrm{V}} \mathrm{O}_{2}$ peak in CHF (Figure 3b). The SOL is a key locomotor and postural muscle in humans and these data, together with a strong correlation between body mass-normalized plantarflexor strength and mass-specific $\dot{\mathrm{V}} \mathrm{O}_{2}$ peak in CHF patients (25), raise the possibility that the SOL may be a sentinel skeletal muscle in terms of identifying the severity of functional impairment in CHF.

These findings were corroborated (23) by observation that the smaller SOL of CHF patients generated lower absolute voluntary active and passive forces (at equivalent levels of passive strain), compared to healthy individuals (Figure 4). Importantly, however, this difference was not present when the data were normalized by muscle cross-sectional area or volume (Figure 4), nor were the normalized active or passive force-length curves different (normalized to optimal fascicle lengths). This detailed single muscle analysis suggested to us that the reduced muscle force in CHF may be more strongly associated with muscle size than muscle structural, mechanical or bioenergetic properties.

Similar findings were observed for the SOL muscle when subjects performed isokinetic contractions over the functional ankle range of motion, suggesting a reduction of the total amount of mechanical work generated by the muscle fibers in CHF (Figure 5a). Nevertheless, isokinetic experiments revealed that the ability of the SOL muscle to produce mechanical work is not compromised in CHF when the data were normalized by muscle volume (Figure 5b). 
Notwithstanding the findings outlined above, which tend to implicate SOL muscle size rather than intrinsic muscle function as a limitation in CHF, we observed some evidence of altered muscle properties in CHF patients. The CHF group exhibited $~ 20 \%(\mathrm{p}<0.05)$ shorter optimal fascicle lengths (i.e. the length where peak voluntary isometric force was achieved) as well as shorter passive fascicle lengths, compared to the age-matched control group (23). The shorter passive fascicle lengths resulted in higher passive force for a given absolute muscle length. It is possible that shorter fascicle lengths result in the muscle operating at longer lengths compared to that of healthy individuals during functional tasks such as walking. This could, in turn, result in altered force-length-velocity characteristics of the muscle that lead to a reduced force and work capacity. Conversely, a shorter optimal fascicle length in CHF might accommodate a more compliant Achilles tendon in CHF; which in turn may result from a smaller tendon cross-sectional area (25). While the precise mechanisms underlying these characteristics have not been defined, they probably reflect a complex interplay between neurohumoral activation, impaired peripheral blood flow and reduced activity levels. For example, impaired peripheral blood flow in CHF may have a more pronounced effect on distal compared with proximal limb muscles, while the greater reduction in SOL muscle volume compared with the medial gastrocnemius may reflect the relative contribution to mechanical work among the lower limb muscles during walking (25). This is an intriguing area which requires further detailed experimentation.

The morphological and functional abnormalities we report above, particularly in the plantarflexors and SOL, could provide a harbinger of impaired function and fatigue (ie initial reduction in the contractile capabilities of the muscle with consequent clinical symptoms of fatigue) early in the clinical course in CHF. In another recent study (24) we compared the mechanics of walking in CHF with that of healthy matched population. Minimal difference in preferred speed or overall joint kinematics and kinetics were observed between groups, a finding that may possibly be explained by optimization of the mechanical cost of transport (i.e. the amount of mechanical work performed per 


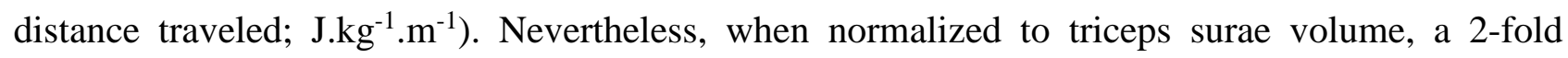
increase in ankle plantarflexion work was required in CHF. This, together with a greater reliance on the ankle to power walking in CHF patients, may contribute to the mechanisms underlying reduced exercise capacity and marked fatigability in CHF.

An important caveat related to the studies described above is that they were undertaken in patients with heart failure associated with impaired ejection fraction. Further studies are required to establish whether a similar relationship exists between aerobic capacity and soleus function in patients with heart failure and preserved ejection fraction.

\section{IMPLICATIONS FOR DESIGN OF EXERCISE INTERVENTIONS}

Studies of exercise training have established that the skeletal muscle limitations to function are amenable to improvement $(7,14,15,17)$ and that exercise training improves functional capacity, cardiovascular mortality (32), hospitalizations (10) and quality of life (35). These benefits typically occur in the absence of changes in cardiac function $(2,26)$. The HF-Action trial, which randomised 2331 heart failure subjects (NYHA II-IV, $\mathrm{EF}<35 \%$ ), indicated that, after adjustment for prognostic factors, exercise training was associated with a 15\% decrease in cardiovascular mortality/CHF hospitalizations. It was concluded that "The HF-ACTION study supports a structured exercise training program for patients with reduced LV function and HF symptoms in addition to evidencebased therapy" (32). However, few attempts have been made to address the question of which exercise prescription will optimise outcomes in HF. Commentary relating to the HF-ACTION trial included "The one thing this trial does not show is what type of exercise to advocate" (32).

Logically, interventions that present the muscle with an intense stimulus, whilst minimizing cardiovascular burden, have the potential to amplify functional gains whilst minimizing acute exercise risk. In accordance with this theoretical framework, we developed in the 1990's 
individually tailored circuit weight training (CWT) programs focusing on isolated muscle groups and based on comprehensive a priori exercise testing $(15,17)$. Because the exercises were performed on isolated muscle groups at any one time, the intensity of effort was relatively high, whilst the cardiovascular burden was modest $(3,13)$. We also successfully established the physiological benefits of such programs in other clinical conditions in a series of experiments $(16,33,34)$. These studies were among the first to utilize resistance modalities in CHF and they directly informed the development of new exercise strategies which are now recognized internationally (27). This work has also directly translated into changes in the management of CHF in Australia and elsewhere (30).

Despite advances in knowledge and clinical practice which have resulted from the development of individually-tailored exercise interventions aimed at reversing physiological limitations in CHF, no previous study has specifically devised exercise training programs based on ameliorating deficits in muscle mechanics (e.g. muscle architecture including muscle length, and mechanical force and work capacities). This is surprising, given the accepted importance in CHF of abnormalities in length tension relationships of cardiac myocytes (i.e. Frank-Starling impacts) and also because of the proven benefits of training interventions focussed on improving muscle mechanics in subjects with other forms of skeletal myopathies (28). The questions that now emerge, include "How can exercise training interventions that specifically target limitations in muscle mechanics be designed for large cohorts of patients, in a manner that is time efficient, tolerable and effective?” This is a future challenge for researchers in the domain of clinical cardiovascular exercise science.

\section{IMPLICATIONS FOR CLINICAL SURVEILLANCE}

Cardiorespiratory fitness ( $\mathrm{VO}_{2}$ peak testing) is routinely assessed in patients with CHF due to its strong prognostic value (18). Such tests are expensive and require a high degree of operator expertise and training. The close relationship observed between soleus muscle function and aerobic 
capacity in patients with CHF makes a case for the serial assessment of soleus muscle function for monitoring changes in clinical status over time. While the studies cited in this review have employed isokinetic dynamometry, protocols to assess soleus muscle strength and endurance that utilise resistance exercise equipment routinely available in cardiac rehabilitation programs could quite easily be developed. Similarly, we have developed non-invasive ultrasound-based approaches to the quantification of the volume of the triceps surae (25) which are cheap and suitable for use in patients with implanted devices, who are MRI contraindicated. Such assessments would logically be conducted by exercise specialists, with results conveyed to medical staff to support clinical surveilance. The degree to which changes in soleus and other skeleletal muscle size and function relate to, or predict, changes in clinical status should form the basis of future research to test and refine such protocols. 


\section{SUMMARY}

Skeletal muscle function is an important locus of the clinical symptoms of fatigue in heart failure, with implications related to survival, stratification for transplant and ventricular assist device implantation. In order to optimise exercise training for subjects with chronic heart failure, the limitations to performance must first be fully understood. Taken together, our recent studies of the skeletal muscle mechanics of patients with CHF suggest that abnormalities in the triceps surae. In particular the SOL appears to be particularly susceptible to the muscle wasting and impaired aerobic capacity which characterize CHF. They also highlight a link between the morphological and functional alterations of the calf muscles and fatigue experienced during walking in CHF. Our recent integrated muscle-level and functional gait analyses indicate that reversing these limitations may depend foremost on restoring muscle size, although muscle functional characteristics (in particular muscle length) may play a further important role. Abnormalities in soleus muscle volume and function may provide a sentinel for detection of early decompensation and a target for specifically designed interventional strategies, although further work is required to develop 3D ultrasound approaches. It is conceivable that, in time, assessment of triceps surae volume and function will become more feasible $\backslash$ and cheaper with less user dependence. We hope that this brief review raises useful hypotheses for future studies aimed at individualised detection of disease progression and the earliest and most effective rehabilitative intervention. 


\section{References}

1 Anker SD, Ponikowski P, Varney S, et al. Wasting as an independent risk factor for mortality in chronic heart failure. Lancet. 1997;349:1050-3.

2 Belardinelli R, Georgiou D, Cianci G, Purcaro A. Randomized, controlled trial of long-term moderate exercise training in chronic heart failure: Effects on functional capacity, quality of life, and clinical outcome. Circulation. 1999;99:1173-82.

3 Cheetham C, Green D, Collis J, Dembo L, O'Driscoll G. Effect of aerobic and resistance exercise on central hemodynamic responses in severe chronic heart failure. J Appl Physiol 2002;93:175-80.

4 Chen J, Normand SL, Wang Y, Krumholz HM. National and regional trends in heart failure hospitalization and mortality rates for Medicare beneficiaries. JAMA. 1998;306:1669-78.

5 Cicoira M, Zanolla L, Franceschini L, et al. Skeletal muscle mass independently predicts peak oxygen consumption and ventilatory response during exercise in noncachectic patients with chronic heart failure. J Am Coll Cardiol. 2001;37:2080-5.

6 Clark AL, Poole-Wilson PA, Coats AJ. Exercise limitation in chronic heart failure: central role of the periphery. J Am Coll Cardiol. 1996;28:1092-102.

7 Coats AJS, Adamopoulos S, Radaelli A, et al. Controlled trial of physical training in chronic heart failure: Exercise performance, hemodynamics, ventilation, and autonomic function. Circulation 1992;85:2119-31.

8 Coats AJS, Clark AL, Piepoli M, Volterrani M, Poole-Wilson PA. Symptoms and quality of life in heart failure: The muscle hypothesis. Br Heart J 1994;72:36-9.

9 Cohn JN, Johnson GR, Shabetai R, et al. Ejection fraction, peak exercise oxygen consumption, cardiothoracic ratio, ventricular arrhythmias, and plasma norepinephrine as determinants of prognosis in heart failure. Circulation 1993;87:5-16.

10 Elahi M, Mahmood M, Shahbaz A, et al. Current concepts underlying benefits of exercise training in congestive heart failure patients. Curr Cardiol Rev 2010;6:104-11. 
11 Fülster S, Tacke M, Sandek A, et al. Muscle wasting in patients with chronic heart failure: results from the studies investigating co-morbidities aggravating heart failure (SICA-HF). Eur Heart J. 2013;34:512-9.

12 Gosker HR, Wouters EF, van der Vusse GJ, Schols AM. Skeletal muscle dysfunction in chronic obstructive pulmonary disease and chronic heart failure: underlying mechanisms and therapy perspectives. Am J Clin Nutr. 2000;71:1033-47.

13 Green DJ, Watts K, Maiorana AJ, O’Driscoll JG. A comparison of ambulatory oxygen consumption during circuit training and aerobic exercise in patients with chronic heart failure. J Cardiopulm Rehab 2001;21:167-74.

14 Hambrecht R, Fiehn E, Weigl C, et al. Regular physical exercise corrects endothelial dysfunction and improves exercise capacity in patients with chronic heart failure. Circulation. 1998;98:2709-15.

15 Maiorana A, O’Driscoll G, Cheetham C, et al. Combined aerobic and resistance exercise training improves functional capacity and strength in CHF. J Appl Physiol. 2000;88:1565-70.

16 Maiorana A, O’Driscoll G, Cheetham C, et al. The effect of combined aerobic and resistance exercise training on vascular function in type 2 diabetes. J Am Coll Cardiol. 2001;38:860-6.

17 Maiorana A, O’Driscoll G, Dembo L, et al. Effect of aerobic and resistance exercise training on vascular function in heart failure. Am J Physiol - Hear Circ Physiol 2000;279:1999-2005.

18 Mancini D, LeJemtel T, Aaronson K. Peak VO2: a simple yet enduring standard. Circulation. 2000;101:1080-2.

19 Mancini DM, Eisen H, Kussmaul W, Mull R, Edmonds LH, Wilson JR. Value of peak exercise oxygen consumption for optimal timing of cardiac transplantation in ambulatory patients with heart failure. Circulation. 1991;83:778-86.

20 Mehra MR, Kobashigawa J, Starling R, et al. Listing criteria for heart transplantation: international society for heart and lung transplantation guidelines for the care of cardiac transplant candidates J Heart Lung Transpl. 2006;25:1024-42. 
21 Miller MS, VanBuren P, LeWinter MM, et al. Chronic heart failure decreases cross-bridge kinetics in single skeletal muscle fibres from humans. J Physiol. 2010;588:4039-53.

22 National. Heart Foundation of Australia and the Cardiac Society of Australia and New Zealand. Guidelines for the prevention, detection and management of chronic heart failure in Australia. 2011.

23 Panizzolo FA, Maiorana A, Naylor LH, et al. Passive and active soleus muscle forces in chronic heart failure in heart failure. Int Soc of Biomechanics XXV Glasgow. 2015.

24 Panizzolo FA, Maiorana AJ, Naylor LH, et al. Gait analysis in chronic heart failure: The calf as a locus of impaired walking capacity. J Biomech. 2014;47:3719-25.

25 Panizzolo FA, Maiorana AJ, Naylor LH, et al. Is the soleus a sentinel muscle for impaired aerobic capacity in heart failure? Med Sci Sports Exerc. 2015;47:498-508.

26 Piepoli MF, Davos C, Francis DP, Coats AJ. ExTraMATCH Collaborative. Exercise training meta-analysis of trials in patients with chronic heart failure (ExTraMATCH). Br Med J. 2004;328:189-92.

27 Pina IL, Apstein CS, Balady GJ, et al. Exercise and Heart Failure: A statement from the American Heart Association Committee on exercise, rehabilitation, and prevention. Circulation. 2003;107:1210-25.

28 Roig M, Shadgan B, Reid WD. Eccentric exercise in patients with chronic health conditions: a systematic review. Physiother Can 2008;60:146-60.

29 Rubenson J, Pires NJ, Loi H, Pinniger GJ, Shannon DG. On the ascent: the soleus operating length is conserved to the ascending limb of the force-length curve across gait mechanics in humans. . J Exp Biol. 2012;215:3539-51.

30 Selig SE, Levinger I, Williams AD, et al. Exercise \& Sports Science Australia Position Statement on exercise training and chronic heart failure. J Sci Med Sport. 2010;13:288-94.

31 Sullivan MJ, Higginbotham MB, Cobb FR. Exercise training in patients with severe left ventricular dysfunction. Hemodynamic and metabolic effects. Circulation. 1988;78:506-15. 
32 TheHeart.org. HF-ACTION misses, but experts say results support intensive exercise in HF patients. http://wwwtheheartorg/article/printdo?primaryKey=919703. 2008.

33 Walsh JH, Bilsborough W, Maiorana A, et al. Exercise training improves conduit vessel function in patients with coronary artery disease. J Appl Physiol. 2003;95:20-5.

34 Walsh JH, Yong G, Cheetham C, et al. Effects of exercise training on conduit and resistance vessel function in treated and untreated hypercholesterolaemic subjects. Eur Heart $J$ 2003;24:1681-9.

35 Willenheimer RE. Exercise training in heart failure improves quality of life and exercise capacity. Eur Heart J 1998;19:774-81. 


\section{Figure captions}

Figure 1. Model depicting the impact of peripheral limitations in chronic heart failure (CHF) based on (6). Abnormalities in skeletal muscle limit exercise capacity and exacerbate symptoms. Personalized exercise prescription is proposed as a means to reverse the progression of the disease by acting on three different pathways influencing skeletal muscle health, namely the muscles' morphological and architectural properties, their mechanical properties, and their functional characteristics during movement.

Figure 2. Schematic showing analytical framework for assessing skeletal muscle morphology, mechanical properties and function including experimental and modeling approaches. Active and passive joint torque and joint range of motion assessed by dynamometry (a). B-mode ultrasound techniques to assess muscle fascicle lengths and pennation angles dynamically (image depicts the soleus muscle) (b); three-dimensional architecture including individual muscle volume and physiological cross-sectional area (image depicts the individual muscles of the triceps surae) (c); and tendon moment arms using a combination of muscle imaging and optical tracking of joint centers (d). Surface electromyography to assess muscle excitation and activation of agonist and antagonist muscles (e). Experimental data (a-e) form the basis for subject-specific musculoskeletal models (OpenSim) (f) that permit further assessment of muscle properties and function including estimates of individual peak active muscle force and passive muscle force.

Figure 3. Triceps surae volume (a) and relationship between SOL muscle volume and aerobic capacity (b). The chronic heart failure (CHF) group is displayed in grey the control group in black; * designates a significant difference $(\mathrm{p}<0.05)$ between the CHF and the control group. 
Figure 4. Absolute peak voluntary force production (a) and normalized absolute peak voluntary force production (b). The chronic heart failure (CHF) group is displayed in grey bars, and the control group in black bars. Data are means \pm S.D.; * designates a significant difference $(\mathrm{p}<0.05)$ between the CHF and the control group.

Figure 5. Work produced by the plantarflexors (a) and work produced by the plantarflexors normalized by SOL muscle volume obtained in isokinetic condition (5\%s) (b). The chronic heart failure (CHF) group is displayed in grey bars, and the control group in black bars. Data are means \pm S.D. This figure was created originally by the authors and has not been previously published. 